\title{
Laparoscopic management of giant hepatic hydatid cyst in a 12-year-old boy: a case report
}

\author{
Banwari lal Bairwa ${ }^{1}$, Aashik Kumar Singh², Shubham Gupta ${ }^{3}$ \\ 'Department of Minimal Access Surgery, MP Birla Hospital and Research Center, Chittorgarh, India \\ ${ }^{2}$ Department of Radiodiagnosis, MP Birla Hospital and Research Center, Chittorgarh, India \\ ${ }^{3}$ Department of Medicine, DR. D. Y. Patil Medical College and Hospital, Kolhapur, India
}

\begin{abstract}
Hydatid disease is a zoonosis caused by a parasite, Echinococcus granulosus, characterized by a cystic lesion in the liver, lung, or (rarely) in other parts of the body. Typical radiological findings make the diagnosis easy and help the surgeons to plan the management of hydatid disease. We report a case of giant hepatic hydatid cyst in a 12-year-old boy who presented with epigastric swelling and pain for 6 months. Contrast-enhanced abdominal computed tomography findings were highly suggestive of a giant hydatid cyst in the left lateral segment of the liver. Laparoscopic partial pericystectomy was done. The patient was discharged on the fifth postoperative day. The patient took oral albendazole preoperatively and postoperatively. Laparoscopic management of hepatic hydatid cysts is safe and effective. Laparoscopic approach is recommended with good technique and controlled suction of cyst content. Surgery combined with oral antihelminthics is the mainstay of treatment for hepatic hydatidosis.
\end{abstract}

Keywords: Laparoscopy, Hydatid cyst, Liver, Child, Case reports
Received June 23, 2021

Revised 1st August 13, 2021 2nd August 24, 2021 3rd September 4, 2021

Accepted September 6, 2021

Corresponding author

Banwari lal Bairwa

Department of Minimal Access Surgery, MP Birla Hospital and Research Center, 18 Udaipur Rd, Maheshpuram, Bapu Nagar Senthri East, Chittorgarh, Rajasthan 312025 , India

Tel: +91-8272836302

E-mail: drbanwaribairwa@gmail.com ORCID:

https://orcid.org/0000-0002-5715-062X

Aashik Kumar Singh and Shubham Gupta contributed equally to this study.

Copyright $\odot$ The Korean Society of Endoscopic and Laparoscopic Surgeons.
This is an Open Access article distributed under the terms of the Creative Commons Attribution Non-Commercial License (http:// creativecommons.org/licenses/by-nc/4.0/) which permits unrestricted non-commercial use, distribution, and reproduction in any medium, provided the original work is properly cited.

\section{INTRODUCTION}

Hydatid disease is an anthropozoonotic disease that affects humans as an accidental host. In humans, slowly enlarging cysts in various organs remain asymptomatic and unnoticed for months to years. Hydatid disease is endemic mainly in Mediterranean countries, the Middle East, the Baltic region, South America, India, northern China, and other countries in which sheep rearing is common and wherein animal husbandry is a common occupation [1].

The liver is the most commonly affected site, followed by the lungs (15\%), spleen (5\%), and other organs ( $2 \%-5 \%)$ [2]. Timely diagnosis and management mitigate complications, such as compression and perforation into adjacent organs or cavities. Chest and abdominal X-ray, ultrasonography, and computed tomography (CT) should identify the characteristic hydatid cyst 
appearance, and the diagnosis is confirmed by detecting specific antibodies via immunodiagnostic testing.

Albendazole, which is an ovicidal, larvicidal, and vermicidal drug, is used for treatment. Surgery is currently the gold-standard treatment for hydatidosis, as it leads reduction in complications, thereby shortening the treatment period. Minimally invasive surgery (laparoscopic and robotics) is safe and feasible, with high adoption rates worldwide. The first laparoscopic treatment of hydatid cyst disease was performed in 1992 [3]. We report a case of giant hydatid cyst of liver which was successfully managed by laparoscopic approach without intraoperative complications.

\section{CASE REPORT}

A 12-year-old boy presented with epigastric swelling and pain for 6 months. The epigastric swelling had been progressively growing. The patient had discomfort and intermittent pain in the epigastric region with no history of fever, vomiting, or jaundice. He had no previous medical, family, and psychosocial history. On clinical examination, a palpable and nontender mass was noted in the epigastric region (Fig. 1). Routine blood investigations, including complete blood count, liver function tests, and renal function tests, were normal, and echinococcal enzyme-linked immunosorbent assay was negative.

Abdominal ultrasonography revealed a large cystic lesion, approximately $13 \mathrm{~cm}$ in diameter, in the left hepatic lobe. For further evaluation, contrast-enhanced CT of the abdomen was conducted, which confirmed a $13 \times 12 \mathrm{~cm}$ cystic lesion in the left lateral segment of the liver. The lesion was compressing the stomach (Fig. 2). Radiological evaluation was highly suggestive of hepatic hydatid disease, and oral albendazole was administered

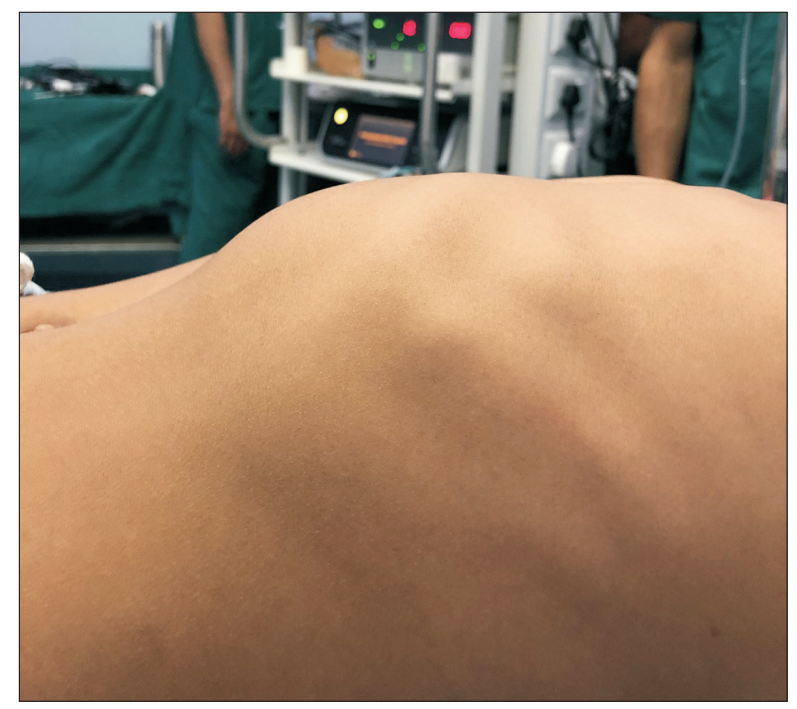

Fig. 1. Preoperative photograph showing the epigastric mass. for 7 days preoperatively.

The patient was planned for laparoscopic Lagrot partial pericystectomy of a giant hepatic hydatid cyst. Pneumoperitoneum was established using a Veres needle through a small infraumbilical incision. A 10-mm trocar was placed in an infraumbilical position. Under laparoscopic guidance (with a $30^{\circ}$ laparoscope), another 5-mm trocar was placed in the right hypochondrium and a $10-\mathrm{mm}$ trocar was placed in the left hypochondrium. Laparoscopic examination revealed only one giant cyst in the left lateral segment of the liver (Fig. 3), and the rest of the intraperitoneal organs were normal. Gauzes soaked in scolicidal agent $(10 \%$ betadine) were placed around the cyst to prevent intraabdominal dissemination in case of spillage. Fluid from the cyst was aspirated, and $50 \mathrm{~mL}$ of scolicidal agent (20\% hypertonic saline) was injected into the cyst and left for 15 minutes to inactivate the parasite. The cyst was then opened, and its contents were aspirated. A partial pericystectomy was done using a harmonic scalpel,

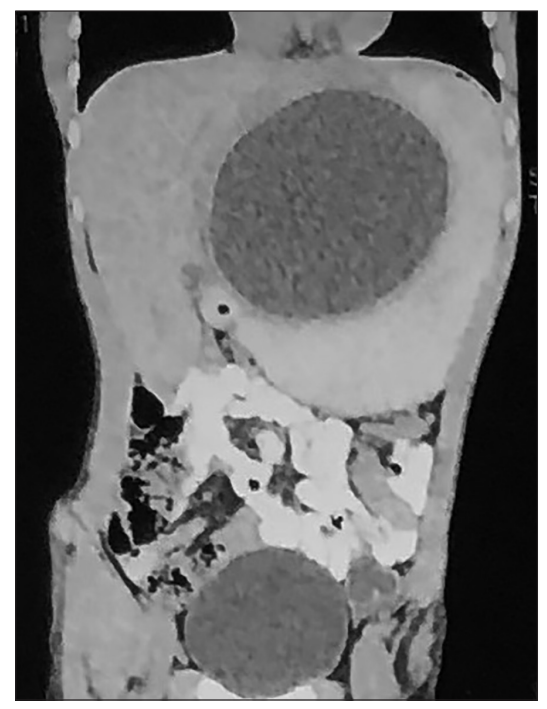

Fig. 2. Contrast-enhanced abdominal computed tomography showing giant a cyst in the left lateral segment of the liver.

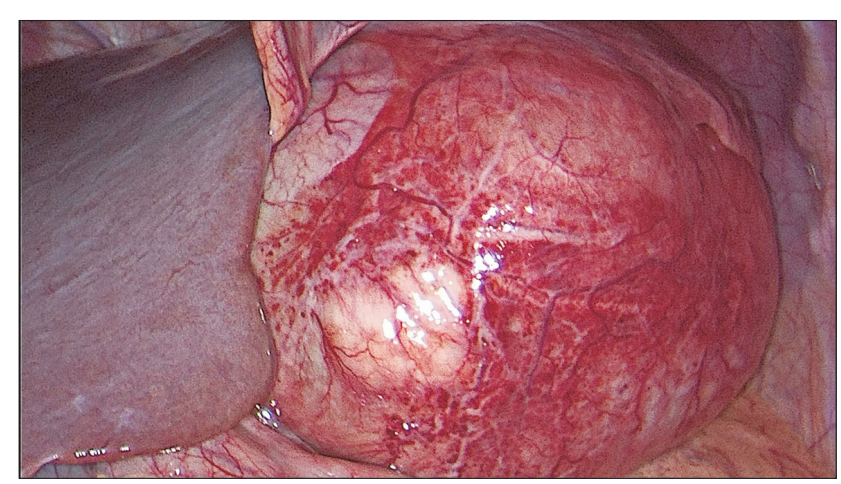

Fig. 3. Laparoscopy image of the giant cyst in the left lateral segment of the liver 


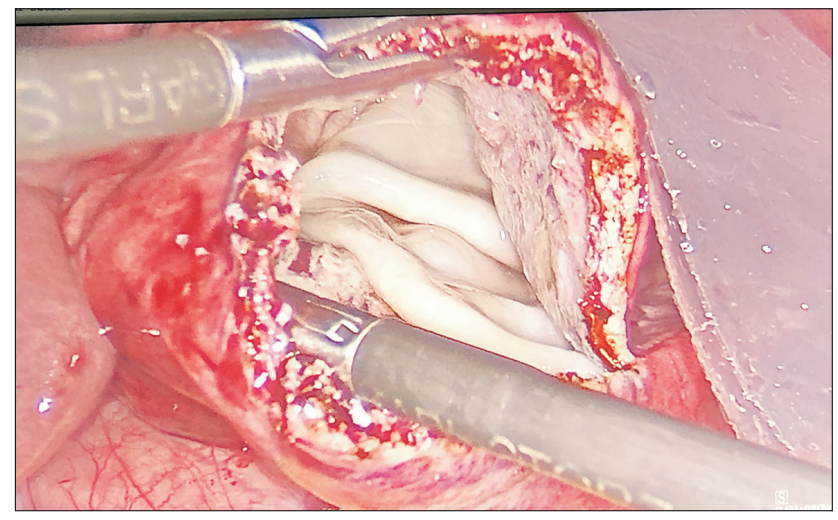

Fig. 4. Intraoperative photograph showing the opened hydatid cyst with the germinal membrane inside.

and around one-third of the cyst wall was excised (Fig. 4). The cystic membrane was retrieved using an Endo Bag (Medtronic, Minneapolis, MN, USA). The remaining cavity was inspected for any bile leakage (Fig. 5), and a 20-French drainage tube was placed inside the remaining cystic cavity. The whole procedure was performed in 55 minutes.

The postoperative period was uneventful. Oral albendazole (10 $\mathrm{mg} / \mathrm{kg} /$ day) in two divided doses was started on postoperative day 1 . No bile leakage was noted in the drainage tube, and the tube was removed on postoperative day 4 . The patient was discharged on the fifth postoperative day.

Histopathological examination of cyst confirmed hydatid disease. Three 4-week cycles of albendazole were completed. Six months postoperatively, no signs of recurrence were present.

\section{DISCUSSION}

Hydatid disease is a zoonotic disease that occurs throughout the world, particularly in those areas where people are involved in animal husbandry. This can involve multiple organs simultaneously. In children, the lungs are usually affected, whereas the liver is commonly affected in adults. Pediatric hepatic hydatid cysts are uncommon [4].

Large cysts are responsible for pressure symptoms in affected organs and have a higher potential for complications. A cyst larger than $5 \mathrm{~cm}$ in diameter is considered a giant cyst. A hydatid cyst basically consists of three layers, namely the adventitia, laminated membrane, and germinal layer. The adventitia is made up of fibrous tissue, which is about 1 to $3 \mathrm{~mm}$ thick and is induced by the host's reaction against the hydatid cyst. Large hepatic hydatid cysts have a higher risk of rupture into the peritoneum and pleural cavity, leading to disseminated disease, cholangitis due to intrabiliary rupture, erosion and rupture into the pericardial space, and severe allergic reaction due to fluid leakage [5].

Small and asymptomatic hepatic hydatid cysts can be man-

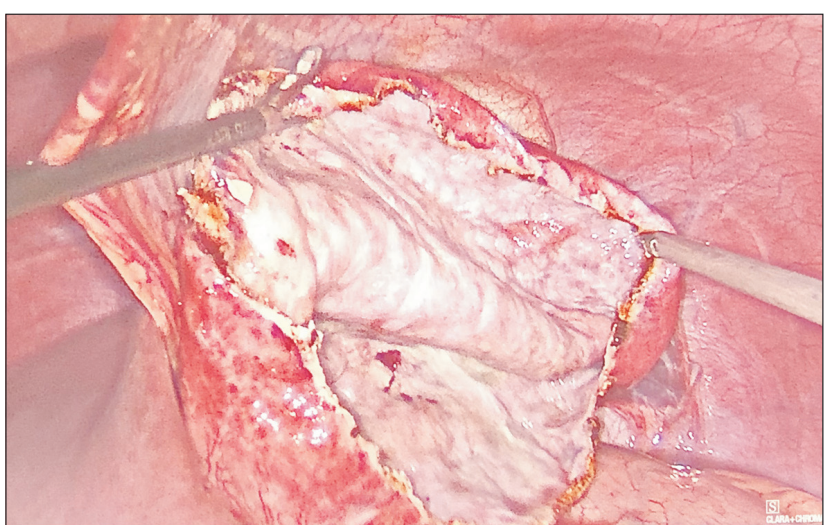

Fig. 5. Intraoperative photograph of the remnant cavity with no signs of bile leakage.

aged with medical treatment, but symptomatic and large cysts need surgical management. Surgery (open or laparoscopy) is the standard treatment. The aims of surgical management are disease cure, decreased morbidity, and prevention of postoperative recurrence. Management of giant liver hydatid disease may constitute a great challenge to attending surgeons, and treatment basically consists of radical or conservative therapies.

In recent times, laparoscopic surgery for hydatid disease is trending. Small incisions, short hospitalization, early recovery, minimal invasion, and better access to multiple and unusual cysts locations are some benefits of laparoscopy over open surgery [6]. Intraperitoneal spillage during laparoscopic surgery is the main potential drawback of laparoscopy due to poor control of suction devices and technical failures [6,7]. Controlled suction of content without spillage lessens the chances of recurrence. A special trocar-cannula system-the Palanivelu hydatid systemenables the removal of an endocyst without excision of the entire cyst [8]. Laparoscopic management of giant hydatid cysts is challenging and has associated limitations and potential complications, such as spillage of cyst content, technical difficulty in cavity obliteration with omentum, and recurrence of disease [9]. Our patient was operated laparoscopically without intraoperative spillage of cyst content. Recently, Acharya et al. [10] developed a single-incision trocar-less endoscopic technique for giant hydatid cyst, which is easily reproducible, avoids intraperitoneal content spillage, allows complete evacuation, and simplifies the obliteration of the residual cavity. Postoperatively, antihelminthics (albendazole) should be given alone or in combination with praziquantel for a minimum of 3 months.

Another modality, which is considered as an alternative approach to surgery in order to minimize recurrence, is the puncture, aspiration, injection, reaspiration (PAIR) approach, which refers to puncturing the cyst, aspirating and then injecting scolicidal agent into the cyst, and then reaspirating. However, PAIR carries the risk of uncontrolled spillage and cyst rupture due to 
the percutaneous nature of the procedure, leading to anaphylactic shock and disease spread. That is why we choose laparoscopic surgery over PAIR for our patient [6]. PAIR is mostly reserved for puncture-accessible cysts, recurrent cysts, cysts unresponsive to medical management, or for when surgery is contraindicated.

The right procedure and the right approach should be individualized for each case. Our patient was managed laparoscopically and recovered well. The presence of large cysts in children can be dangerous, as it might affect the growth of other developing organs. The most common morbidity following hydatid liver surgery is biliary fistula formation [9].

Laparoscopic management of giant hepatic hydatid cysts is minimally invasive, efficient, and effective. Laparoscopic or open surgical intervention with antihelminthics is the treatment strategy of choice for hydatid disease.

\section{NOTES}

\section{Ethical statements}

We developed this case report in compliance with the principles of the Declaration of Helsinki. The work was reviewed and approved by the Institutional Review Board of MP Birla Hospital and Research Center (No. MPBHRC/2021/02). Written informed consent was obtained.

\section{Authors' contributions}

Conceptualization, Data curation: BLB, AKS

Formal analysis, Investigation, Methodology: All authors

Project administration, Visualization: BLB

Writing-original draft: All authors

Writing-review \& editing: BLB

All authors read and approved the final manuscript.

\section{Conflict of interest}

All authors have no conflicts of interest to declare.

\section{Funding/support}

None.

\section{ORCID}

Banwari lal Bairwa, https://orcid.org/0000-0002-5715-062X

Aashik Kumar Singh, https://orcid.org/0000-0003-3416-7223

Shubham Gupta, https://orcid.org/0000-0002-8495-413X

\section{REFERENCES}

1. Shrestha AL, Paudel SB, Malla SK. Multiple giant liver cysts in a nepalese lad. Case Rep Pediatr 2020;2020:6196252.

2. Safioleas M, Stamoulis I, Theocharis S, Moulakakis K, Makris S, Kostakis A. Primary hydatid disease of the gallbladder: a rare clinical entity. J Hepatobiliary Pancreat Surg 2004;11:352-356.

3. Stanciulea O, Eftimie MA, Mosteanu I, Tirca L, Popescu I. Primary giant splenic and hepatic echinococcal cysts treated by laparoscopy. J Minim Invasive Surg 2017;20:155-159.

4. Jairajpuri ZS, Jetley S, Hassan MJ, Hussain M. Hydatid disease in childhood: revisited report of an interesting case. J Parasit Dis 2012;36:265-268

5. Sabzevari S, Badirzadeh A, Shahkaram R, Seyyedin M. Traumatic rupture of liver hydatid cysts into the peritoneal cavity of an 11-yearold boy: a case report from Iran. Rev Soc Bras Med Trop 2017;50:864867.

6. Hemmati SH. How to build a simple and safe laparoscopic hydatid evaluation system. JSLS 2014;18:e2014.00314.

7. Ali FM. Laparoscopic versus open management of hydatid cyst of liver. World J Laparosc Surg 2011;4:7-11.

8. Palanivelu C, Senthilkumar R, Jani K, et al. Palanivelu hydatid system for safe and efficacious laparoscopic management of hepatic hydatid disease. Surg Endosc 2006;20:1909-1913.

9. Vennarecci G, Manfredelli S, Guglielmo N, Laurenzi A, Goletti D, Ettorre GM. Major liver resection for recurrent hydatid cyst of the liver after suboptimal treatment. Updates Surg 2016;68:179-184.

10. Acharya H, Agrawal V, Tiwari A, Sharma D. Single-incision trocarless endoscopic management of giant liver hydatid cyst in children. J Minim Access Surg 2018;14:130-133. 